Pacific Journal of Mathematics

OSCILLATION RESULTS FOR A NONHOMOGENEOUS 


\title{
OSCILLATION RESULTS FOR A NONHOMOGENEOUS EQUATION
}

\author{
SAMUEL M. RANKIN, III
}

The purpose of this note is to investigate oscillatory properties of solutions of the equation

$$
y^{\prime \prime}+p(t) y=f(t)
$$

via the transformation $y(t)=u(t) z(t)$ where $u(t)$ is a solution of the equation

$$
u^{\prime \prime}+p(t) u=0 \text {. }
$$

Equation (2) is assumed to be nonoscillatory throughout the paper. This represents a distinct change from most of the recent work concerning oscillation in equation (1).

The transformation $y(t)=\phi(t) z(t)$ transforms equation (1) into

$$
\left(\phi^{2} z^{\prime}\right)^{\prime}+\phi(t)\left(\phi^{\prime \prime}(t)+p(t) \phi(t)\right) z=f(t) \phi(t) .
$$

If $\phi(t)$ is a solution of (2) then (3) becomes

$$
\left(\phi^{2} z^{\prime}\right)^{\prime}=f(t) \phi(t) \text {. }
$$

Equation $\left(3^{\prime}\right)$ enables us to characterize the oscillatory behavior of solutions of (1) in terms of the forcing function $f(t)$ and the nonoscillatory solutions of equation (2). The need for "explicit" sign conditions on $p(t)$ is eliminated. However, some implicit sign conditions will be assumed, that is, the solution $\phi(t)$ of equation (2) will be given properties that are implied by specific sign conditions on $p(t)$.

In recent articles Macki [10] and Komkov [7] have pointed out the usefulness of the transformation $u(t)=\phi(t) z(t)$ in studying qualitative properties of the differential equation

$$
\left(r(t) u^{\prime}\right)^{\prime}+p(t) u=0 .
$$

As usual a nontrivial solution $y(t)(u(t))$ of equation (1) [resp. (2)] is oscillatory if on each ray $(a, \infty)(a>0)$ there exists a $t_{0} \in(a, \infty)$ with $y\left(t_{0}\right)=0\left(u\left(t_{0}\right)=0\right)$. Equation (1) [resp. (2)] is oscillatory if all solutions are oscillatory. A solution $y(t)$ [resp. $u(t)$ ] of equation (1) [resp. (2)] is nonoscillatory if it is eventually nonzero. It is well known that all solutions of equation (2) are either oscillatory or nonoscillatory. The functions $p(t)$ and $f(t)$ are assumed to be continuous on $[0, \infty)$, so only solutions on the interval $[0, \infty)$ will be 
considered.

There has been considerable interest in the oscillatory properties of equation (1) and some of its nonlinear analogues, for example, Abramovich [1], Grimmer and Patula [2], Graef and Spikes [3] [4], Hammett [5], Jones and Rankin [6], Lovelady [8] [9], Rankin [11] [12], Singh [13], Skidmore and Bowers [14], Tefteller [15] and Wallgren [16]. In each of these papers, except [2] and [11], a sign condition is imposed on $p(t)$, and in all but [6] and [8] the unforced equation is either implicitly or explicitly assumed oscillatory.

To motivate our first theorem, consider the following examples:

EXAMPLE 1. $u^{\prime \prime}+(1 / 4) t^{-2} u=0 y^{\prime \prime}+(1 / 4) t^{-2} y=t(1 / 2) \sin t$ and

EXAMPLE 2. $u^{\prime \prime}=0 \quad y^{\prime \prime}=t \sin t$.

It is seen below that the nonhomogeneous equations in the above examples are oscillatory.

THEOREM 1. If there exists a positive solution $\phi(t)$ of equation (2) such that for each $T>0$ and for some $M>0$

(i ) $\varliminf_{t \rightarrow \infty} \int_{T}^{t} f(s) \dot{\varphi}(s) d s=-\infty$ and $\varlimsup_{t \rightarrow \infty} \int_{T^{\prime}}^{t} f(s) \dot{\varphi}(s) d s=\infty$,

(ii ) $\left|\int_{T}^{t} 1 / \dot{\phi}^{2}(s) \int_{T}^{s} f(r) \dot{\varphi}(r) d r \cdot d s\right| \leqq M \int_{T}^{t} d s / \phi^{2}(s)$ and

(iii) $\lim _{t \rightarrow \infty} \int_{T}^{t} d s / \dot{\phi}^{2}(s)=\infty$, then equation (1) is oscillatory.

REMARK. In Theorem 1 and the theorems given below, it is easily seen that if $f(t)$ satisfies our hypothesis, so does $-f(t)$. The transformation $v=-y$ changes (1) into an equation of the same form preserving the assumptions of the theorems. Therefore, when we assume a solution $y(t)$ of equation (1) is nonoscillatory, we will assume $y(t)>0$ on some ray $(a, \infty)$.

Proof of Theorem 1. Suppose equation (1) is nonoscillatory so that there exists a solution $y(t)$ of equation (1) such that $y(t)>0$ on $(a, \infty)$ for some $a>0$. The function $z(t)$, defined by $y(t)=$ $\phi(t) z(t)$, is a nonoscillatory solution of equation $\left(3^{\prime}\right)$. After integrating $\left(3^{\prime}\right)$ and applying (i), we have that $\varliminf_{t \rightarrow \infty} \dot{\phi}^{2}(t) z^{\prime}(t)=-\infty$. Now choosing $T_{1}>T$ such that $\dot{\phi}^{2}\left(T_{1}\right) z^{\prime}\left(T_{1}\right)<-2 M$, we have by integration that

$$
z(t)=z\left(T_{1}\right)+\dot{\phi}^{2}\left(T_{1}\right) z^{\prime}\left(T_{1}\right) \int_{T_{1}}^{t} d s / \phi^{2}(s)+\int_{T_{1}}^{t} 1 / \phi^{2}(s) \int_{T_{1}}^{s} f(r) \dot{\phi}(r) d r d s .
$$


From (ii) we obtain

$$
z(t)<z\left(T_{1}\right)-M \int_{T_{1}}^{t} d s / \phi^{2}(s),
$$

and by (iii) the solution $z(t)$ is eventually negative. This contradicts $y(t)>0$ on $[T, \infty)$.

REMARK. In Example (1), choose $\dot{\phi}(t)=t^{1 / 2}$ and in Example (2), $\phi(t)=1$.

THEOREM 2. If there exists a positive solution $\phi(t)$ of equation (2) such that for $T$ sufficiently large

(i ) $\lim _{t \rightarrow \infty} \int_{T}^{t} 1 / \phi^{2}(s) \int_{T}^{s} f(r) \phi(r) d r d s=-\infty$ and $\varlimsup_{t \rightarrow \infty} \int_{T}^{t} 1 / \phi^{2}(s) \int_{T}^{s} f(r) \phi(r) d r d s=\infty$ and

(ii) $\lim _{t \rightarrow \infty} \int_{T}^{t} d s / \dot{\phi}^{2}(s)<\infty$ then equation (1) is oscillatory.

Proof. Suppose there exists a solution $y(t)$ of equation (2) such that $y(t)>0$ on $(a, \infty)$ for some $a>0$, then the function $z(t)$, defined by $y(t)=\phi(t) z(t)$, is a positive solution of equation $\left(3^{\prime}\right)$ on $[T, \infty)$ for some $T>a$. Integrating equation $\left(3^{\prime}\right)$ twice we have

$$
z(t)=z(T)+\phi^{2}(T) z^{\prime}(T) \int_{T}^{t} d s / \dot{\phi}^{2}(s)+\int_{T}^{t} 1 / \phi^{2}(s) \int_{T}^{s} f(r) \dot{\phi}(r) d r d s .
$$

By conditions (i) and (ii), $z(t)$ satisfies $z\left(t_{0}\right)<0$ for some $t_{0}>T$, thus contradicting the positivity of $y(t)$ on $(a, \infty)$.

EXAMPLE 3. The equation $y^{\prime \prime}-y=e^{3 t} \sin t$ illustrates Theorem 2 where $\phi(t)=e^{t}$. Also for $y^{\prime \prime}=t^{3} \cos t$ choose $\phi(t)=t$.

EXAMPLE 4. For the equation $y^{\prime \prime}-y=\sin t$ all of the conditions of Theorems 1 and 2 are not met. This equation has the general solution $y(t)=-1 / 2 \sin t+c_{1} e^{-t}+c_{2} e^{t}$. Notice that all bounded solutions on $[0, \infty)$ can be written in the form $y(t)=-$ $1 / 2 \sin t+c_{1} e^{-t}$ for some $c_{1}$. It is easily seen that these solutions are oscillatory. The following theorem can now be stated.

THEOREM 3. If there exists a positive bounded solution $\phi(t)$ of equation (2) and an $a>0$ such that

(i ) $\lim _{t \rightarrow \infty} \phi(t) \int_{T}^{t} d s / \phi^{2}(s)=\lim _{t \rightarrow \infty} \int_{T}^{t} d s / \phi^{2}(s)=\infty$ for each $T>a$ and

(ii) there exists a sequence $\left\{T_{n}\right\}_{n=1}^{\infty}$ such that $\lim _{n \rightarrow \infty} T_{n}=\infty$, 
$\lim _{t \rightarrow \infty} \int_{T_{n}}^{t} f(s) \dot{\phi}(s) d s=0, \lim _{t \rightarrow \infty} \int_{T_{n}}^{t} 1 / \phi^{2}(s) \int_{T_{n}}^{s} f(r) \dot{\phi}(r) d r d s=-\infty, \varlimsup_{t \rightarrow \infty} \int_{T_{n}}^{t} 1 / \phi^{2}(s)$ $\int_{T_{n}}^{s} f(r) \dot{\phi}(r) d r d s=\infty$, and $\left|\phi(t) \int_{T_{n}}^{t} 1 / \phi^{2}(s) \int_{T_{n}}^{s} f(r) \dot{\phi}(r) d r d s\right|$ is bounded, then all bounded solutions of equation (1) are oscillatory.

Proof. Suppose there exists a bounded solution $y(t)$ of equation (1) such that $y(t)>0$ on $[T, \infty)(T>a)$. Integrating equation $\left(3^{\prime}\right)$ from $T_{n}$ to $t$ for some $T_{n}>T$, we have

$(*) \quad \phi^{2}(t) z^{\prime}(t)=\phi^{2}\left(T_{n}\right) z^{\prime}\left(T_{n}\right)+\int_{T_{n}}^{t} f(s) \dot{\phi}(s) d s$.

$\phi^{2}\left(T_{n}\right) z^{\prime}\left(T_{n}\right)$ is greater than 0 , for each $n$, for if $\phi^{2}\left(T_{n}\right) z^{\prime}\left(T_{n}\right)=0$, a second integration yields

$$
z(t)=z\left(T_{n}\right)+\int_{T_{n}}^{t} 1 / \phi^{2}(s) \int_{T_{n}}^{s} f(r) \phi(r) d r d s \text { and by (ii) }
$$

$\underline{\lim } z(t)=-\infty$, a contradiction. If $\phi^{2}\left(T_{n}\right) z^{\prime}\left(T_{n}\right)$ is negative, then choose $\varepsilon>0$ such that $\phi^{2}\left(T_{n}\right) z^{\prime}\left(T_{n}\right)+\varepsilon<0$. By (ii), it is true for $t>T^{\prime}$ for some $T^{\prime}>T_{n}$ that $\int_{T_{n}}^{t} f(s) \dot{\phi}(s) d s<\varepsilon$ and from $\left(^{*}\right) z^{\prime}(t)<$ $\phi^{2}\left(T_{n}\right) z^{\prime}\left(T_{n}\right)+\varepsilon / \phi^{2}(t)$, for $t \geqq T^{\prime}$. Integrating the above inequality from $T^{\prime}$ to $t$ gives $z(t)<\left(\phi^{2}\left(T_{n}\right) z^{\prime}\left(T_{n}\right)+\varepsilon\right) \int_{T_{n}}^{t} d s / \phi^{2}(s)+z\left(T^{\prime}\right)$. Applying (i), it can be seen that $z(t)$ will eventually be negative.

Now, integrating (*) from $T_{n}$ to $t$ and multiplying by $\phi(t)$ gives

$$
\begin{aligned}
y(t)=\dot{\phi}(t) z(t)=\phi(t) z\left(T_{n}\right) & +\dot{\phi}^{2}\left(T_{n}\right) z^{\prime}\left(T_{n}\right) \dot{\phi}(t) \int_{T_{n}}^{t} d s / \dot{\phi}^{2}(s) \\
& +\dot{\phi}(t) \int_{T_{n}}^{t} 1 / \dot{\phi}^{2}(s) \int_{T_{n}}^{s} f(r) \dot{\phi}(r) d r d s .
\end{aligned}
$$

The left side of the above equality remains bounded while the right side approaches infinity by (i), (ii), and the fact that $\dot{\phi}^{2}\left(T_{n}\right) z^{\prime}\left(T_{n}\right)>0$; the theorem is proved.

It is an easy exercise to see that $w(t)=y_{1}(t)-y_{2}(t)$ is a solution of equation (2) whenever $y_{1}(t)$ and $y_{2}(t)$ are solutions of equation (1). Thus if equation (2) is nonoscillatory, there are at most a finite number of points $t_{1} \cdots t_{n}$ such that $y_{1}\left(t_{i}\right)=y_{2}\left(t_{i}\right)$ for $i=$ $1,2, \cdots, n$. Let us further assume that $y_{1}(t)$ and $y_{2}(t)$ have no double zeros for large $t$ and that for sufficiently large $a, b, y_{1}(a)=y_{1}(b)=0$ with $y_{1} \neq 0$ on $(a, b)$. Then if $y_{2}\left(t_{0}\right)=0$ for some $t_{0} \in(a, b)$, the solution $y_{2}(t)$ of (1) has an even number of zeros in $(a, b)$.

To obtain asymptotic results for nonoscillatory solutions of equation (1), equation (3) is considered once more where $\dot{\phi}(t)$ is not 
necessarily a solution of equation (2). The following results of Hammett [5] and Graef and Spikes [3] for the differential equation

$$
\left(r(t) v^{\prime}\right)^{\prime}+p(t) v=f(t)
$$

will be useful.

THEOREM 4. [Hammett, 5]. If

(i ) $r(t)>k>0$ on $[0, \infty)$ and $\int_{0}^{\infty} d t / r(t)=\infty$,

(ii) $p(t)>k>0$

(iii) $f \in L(0, \infty)$

then all nonoscillatory solutions $v(t)$ of $(4)$ satisfy $\lim v(t)=0$.

THEOREM 5. [Graef and Spikes, 3]. If

(i ) $r(t)>0$ on $[0, \infty)$ and $\int_{0}^{\infty} d t / r(t)=\infty$,

(ii) $p(t)>0$ and $\int_{0}^{\infty} p(s) d s=\infty$,

(iii) $\int_{0}^{\infty}\left(\int_{0}^{w} d s / r(s)\right)|f(w)| d w<\infty$,

then all nonoscillatory solutions $v(t)$ of (4) satisfy $\lim _{t \rightarrow \infty} v(t)=0$.

THEOREM 6. If there exists a positive function $\phi(t)$ such that $\phi(t) f(t) \in L(0, \infty), \quad \phi\left(\phi^{\prime \prime}(t)+p(t) \phi(t)\right)>K_{1} \phi^{2}(t)>K_{1}$ for some $K_{1}>0$ and $\int^{\infty} d s / \phi^{2}(s)=\infty$, then every nonoscillatory solution of equation (1) satisfies $\lim _{t \rightarrow \infty} y(t) \phi(t)=0$.

Proof. By Theorem 4 and the hypothesis, each nonoscillatory solution $z(t)$ of equation (3) satisfies $\lim _{t \rightarrow \infty} z(t)=0$.

EXAMPLE 5. For the equation

$$
y^{\prime \prime}+t^{-1} y=2 t^{-3}+t^{-2}
$$

let $\phi(t)=t^{1 / 2}$ and the conditions of the theorem are satisfied. Notice that equation (5) does not satisfy all of Hammett's hypothesis.

THEOREM 7. If $\int_{b}^{\infty}\left(\int_{b}^{s} d w / \phi^{2}(w)\right)|\phi(s) f(s)| d s<\infty$ where $\phi(t)>0$, $\int^{\infty} d w / \phi^{2}(w)=\infty, \int^{\infty}\left[\phi^{\prime \prime}(t) \phi(t)+p(t) \phi^{2}(t)\right] d t=\infty$, and $\phi^{\prime \prime} \phi+p(t) \phi^{2}>0$ then all nonoscillatory solutions of equation (1) satisfy $\lim y(t) / \phi(t)=0$.

Proof. Equation (3) now satisfies the hypothesis of Theorem 5 and so $\lim _{t \rightarrow \infty} z(t)=0$ for each nonoscillatory solution $z(t)$ of equation (3). 
EXAMPLE 6. The following equation is more general than equation (1) but illustrates the usefulness of the transformation $y(t)=$ $\phi(t) z(t)$ :

$$
\left(t y^{\prime}\right)^{\prime}+t^{-1 / 2} y=t^{-2}+t^{-3 / 2}
$$

Equation (6) does not satisfy condition (iii) of Theorem 5. However, using the above transformation with $\phi(t)=t^{-1 / 4}$, all conditions of Graef and Spikes' theorem are satisfied for the equation

$$
\left(t^{1 / 2} z^{\prime}\right)^{\prime}+\left(5 / 16 t^{-10 / 4}+t^{-1}\right) z=t^{-9 / 4}+t^{-7 / 4}
$$

and so for all nonscillatory solutions $z(t), \lim _{t \rightarrow \infty} z(t)=0$. Since $y(t)=$ $t^{-1 / 4} z(t)$, all nonoscillatory solutions $y(t)$ of equation (6) satisfy $\lim _{t \rightarrow \infty} t^{1 / 4} y(t)=0$.

REMARK. The transformation $y(t)=\phi(t) z(t)$ maks it possible not to require $p(t)$ to be positive as required in [3] and [5].

ACKNOWLEDGMENT. The author wishes to thank the referee for his many helpful comments and suggestions.

\section{REFERENCES}

1. S. Abramovich, On the behavior of the solutions of $y^{\prime \prime}+p(t)=f(t)$, J. Math. Anal. Appl., 52 (1975), 465-470.

2. R. Grimmer and W. Patula, Nonoscillatory solutions of forced second order linear equations, J. Math. Anal. Appl., 56 (1976), 452-459.

3. J. Graef and P. Spikes, Asymptotic behavior of solutions of a second order nonlinear differential equation, J. Differential Equations, 17 (1975), 461-476.

4. - Sufficient conditions for nonoscillation of a second order nonlinear differential equation, Proc. Amer. Math. Soc., 50 (1975), 289-292.

5. M. Hammett, Nonoscillation properties of a nonlinear differential equation, Proc. Amer. Math. Soc., 30 (1971), 92-96.

6. G. Jones and S. Rankin, Oscillation of a forced non-linear second order differential equation, in Accademia Nazionale Dei Lencei, 59 (1975), 702-705.

7. V. Komkov, A technique for the detection of oscillation of second order ordinary differential equations, Pacific J. Math., 42 (1972), 105-115.

8. D. Lovelady, Oscillations induced by forcing functions, to appear Accademia Nazionale dei Lencei.

9. - Oscillation and a class of forced even order differential equations, to appear.

10. J. Macki, Application of change of variables in the qualitative theory of second order linear ordinary differential equations, SIAM Review, 18 (1976), 269-274.

11. S. Rankin, Oscillation for second order nonhomogeneous linear differential equations, J. Math. Anal. Appl., 53 (1976).

12. - Oscillation of a forced second order nonlinear differential equation, Proc. Amer. Math. Soc., 58 (1976).

13. B. Singh, Comparative study of asymptotic nonoscillation and quick oscillation of second order linear differential equations, J. Math. and Phy. Sci., 8 (1974), 363-376. 
14. A. Skidmore and J. Bowers, Oscillatory behavior of solutions of $y^{\prime \prime}+p(t) y=f(t)$, J. Math. Anal. Appl., 49 (1975), 317-323.

15. S. Tefteller, Oscillation of second order nonhomogeneous linear differential equations, SIAM J. Applied Math., 31 (1976), 461-467.

16. T. Wallgren, Oscillation of solutions of the differential equation $y^{\prime \prime}+p(t) y=f(t)$, SIAM J. Math. Anal., 7 (1976), 848-857.

Received June 9, 1976 and in revised form October 18, 1978.

MURRay State UnIVERSity

MURRAY, KY 42071

Current Address: Department of Mathematics, West Virginia University, Morgantown, West Virginia 26506 



\section{PACIFIC JOURNAL OF MATHEMATICS}

\section{EDITORS}

RICHARD ARENS (Managing Editor)

University of California

Los Angeles, California 90024

C. W. CURTIS

University of Oregon

Eugene, OR 97403

C. C. MOORE

University of California

Berkeley, CA 94720
J. DugundjI

Department of Mathematics University of Southern Californı Los Angeles, California 90007

R. Finn and J. Milgram Stanford University Stanford, California 94305

ASSOCIATE EDITORS

E. F. BECKENBACH

B. H. NeUmanN

F. WOLF

K. YoSHIDA

\section{SUPPORTING INSTITUTIONS}

UNIVERSITY OF BRITISH COLUMBIA CALIFORNIA INSTITUTE OF TECHNOLOGY

UNIVERSITY OF CALIFORNIA

MONTANA STATE UNIVERSITY

UNIVERSITY OF NEVADA, RENO

NEW MEXICO STATE UNIVERSITY

OREGON STATE UNIVERSITY

UNIVERSITY OF OREGON
UNIVERSITY OF SOUTHERN CALIFORNIA

STANFORD UNIVERSITY

UNIVERSITY OF HAWAII

UNIVERSITY OF TOKYO

UNIVERSITY OF UTAH

WASHINGTON STATE UNIVERSITY

UNIVERSITY OF WASHINGTON 


\section{Pacific Journal of Mathematics}

\section{Vol. 80, No. $1 \quad$ September, 1979}

Jeroen Bruijning and Jun-iti Nagata, A characterization of covering dimension by

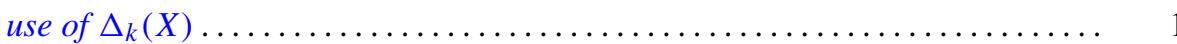

John J. Buoni and Albert Jonathan Klein, On the generalized Calkin algebra ...... 9

Thomas Ashland Chapman, Homotopy conditions which detect simple homotopy

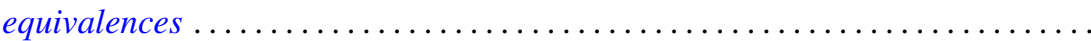

John Albert Chatfield, Solution for an integral equation with continuous interval

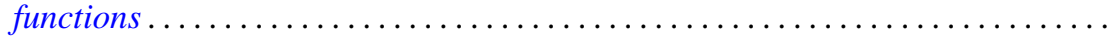

Ajit Kaur Chilana and Ajay Kumar, Spectral synthesis in Segal algebras on

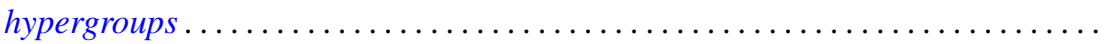

Lung O. Chung, Jiang Luh and Anthony N. Richoux, Derivations and

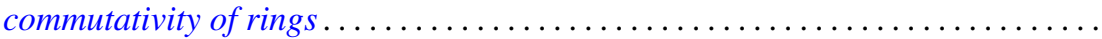

Michael George Cowling and Paul Rodway, Restrictions of certain function spaces to closed subgroups of locally compact groups .....................

David Dixon, The fundamental divisor of normal double points of surfaces........

Hans Georg Feichtinger, Colin C. Graham and Eric Howard Lakien,

Nonfactorization in commutative, weakly selfadjoint Banach algebras . . . . . . .

Michael Freedman, Cancelling 1-handles and some topological imbeddings ....... .

Frank E., III Gerth, The Iwasawa invariant $\mu$ for quadratic fields . . . . . . . . . . . . . .

Maurice Gilmore, Three-dimensional open books constructed from the identity

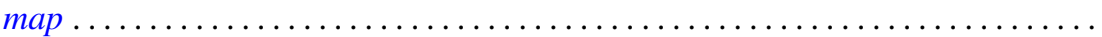

Stanley P. Gudder, A Radon-Nikodým theorem for $*$-algebras .

Peter Wamer Harley, III and George Frank McNulty, When is a point Borel? .

Charles Henry Heiberg, Fourier series with bounded convolution powers . .

Rebecca A. Herb, Characters of averaged discrete series on semisimple real Lie

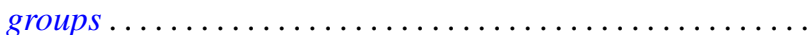

Hideo Imai, On singular indices of rotation free densities . .

Sushil Jajodia, On 2-dimensional CW-complexes with a single 2-cell . . .

Herbert Meyer Kamowitz, Compact operators of the form $u C_{\varphi}$

Matthew Liu and Billy E. Rhoades, Some properties of the Chebyshev method...

213

George Edgar Parker, Semigroups of continuous transformations and generating

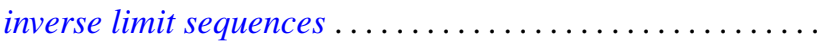

Samuel Murray Rankin, III, Oscillation results for a nonhomogeneous

equation ...

Martin Scharlemann, Transverse Whitehead triangulations ...

Gary Joseph Sherman, A lower bound for the number of conjugacy classes in a

finite nilpotent group

Richard Arthur Shoop, The Lebesgue constants for $\left(f, d_{n}\right)$-summability .

Stuart Jay Sidney, Functions which operate on the real part of a uniform

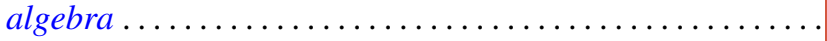

Tim Eden Traynor, The group-valued Lebesgue decomposition 